\title{
Investigación aplicada al territorio desde la Medicina
}

\section{Familiar}

\section{Research applied to the territory from Family Medicine \\ Pesquisa aplicada ao território desde a Medicina Familiar}

\section{Grupo de Trabajo:}

Ciuffolini María Beatriz (Argentina); Rodríguez Morales Victoria (Bolivia); Magne Anzoleaga Jenny (Bolivia); Brito Rodríguez Angelo (Brasil); Gomes da Trindade Thiago (Brasil); Rodríguez Batista Sandro (Brasi); Tajra Fabio Solon (Brasil);Wegner Falk João (Brasil); Alarcón José Domingo (Colombia); Bejarano Liliana (Colombia); Castrillón Ricardo (Colombia); Castro Florez Ximena (Colombia); Criollo Claudia Patricia (Colombia); Garcia Lina María (Colombia); Garzón Andrea del Pilar (Colombia); Hamman Echeverri Otto (Colombia); Hernández Marcela (Colombia); Parra Luis Gabriel (Colombia); Malagón Rafael ( Colombia); Urdinola Cuellar Stella (Colombia); González Reyes Carolina (Chile); Vargas Patricia (Chile); Orellana Navarrete Lusy Paulyna ( Ecuador); Pons Alvarez Octavio Noel (México); Rubio Florez Erik Noe (México); Martínez Cabrera Roberto (Nicaragua); Camacho Cinthia (Paraguay); Riveros Myriam (Paraguay); Villanueva Rosa (Perú); García Martinez Eldon (República Dominicana).

\section{Resumen}

En el contexto la VII Cumbre Iberoamericana de Medicina Familiar, efectuada en Cali, Colombia el 13 y 14 de Marzo de 2018, el grupo de investigación y la Red Iberoamericana de Medicina Familiar (IBIMEFA) con el objetivo de Identificar las percepciones de médicos familiares graduados y en proceso de formación de Iberoamérica, sobre las condiciones que tienen para desarrollar investigación aplicada al territorio, realizaron un estudio exploratorio, descriptivo, de corte transversal en los países miembros de la Confederación Iberoamericana de Medicina Familiar (CIMF). Para la recolección de información, se diseñó una encuesta con 51 items, cuyo contenido fue validado por el grupo de trabajo. El instrumento se almacenó en Google Drive y su divulgación se efectuó a través de medios virtuales. Se obtuvo respuesta de 277 personas, representantes de 16 países de Iberoamérica en los que se encontró que existe interés y potencial dentro del grupo de la Red IBIMEFA, para realizar estudios con el enfoque de investigación aplicada al territorio, aunque se deben superar dificultades de tiempo y financiación para lograrlo, así como encontrar estrategias que permitan hacer un trabajo cooperativo que consolide la Red.

Palabras clave: Medicina familiar y Comunitaria; Determinantes sociales; Territorio; Investigación

Cómo citar: Rodríguez MA, Mahecha LM, Flores O, Fernández MA. Investigación aplicada al territorio desde la Medicina Familiar. Rev Bras Med Fam Comunidade. 2018;13(Suppl 1):29-42. http://dx.doi.org/10.5712/rbmfc13(40)1851

\author{
Mauricio Alberto Rodríguez Escobara \\ Lina María Mahecha Rivera ${ }^{b}$ \\ Omaira Flores Martíne ${ }^{c}$ \\ Miguel Ángel Fernández Ortega ${ }^{d}$
}

a Universidad El Bosque; Sociedad Colombiana de Medicina Familiar (SOCMEF). Bogotá, Colombia.

medicinafamiliar@unbosque.edu.co (Autor correspondiente)

b Universidad de Ciencias Aplicadas y Ambientales (UDCA); Sociedad Colombiana de Medicina Familiar (SOCMEF). Bogotá, Colombia. limahecha@udca.edu.co

c Instituto Venezolano de los Seguros Sociales (IVSS); Colegio Universitario de Enfermería del Centro Médico (CUECMC); Sociedad Venezolana de Medicina Familiar (SOVEMEFA). Caracas, Venezuela. omairaflores@yahoo.com

d Facultad de Medicina, Universidad Nacional Autónoma de México (UNAM); Confederación Iberoamericana de Medicina Familiar (WONCA-Iberoamericana-CIMF). México. miguelaf03@live.com

\author{
Financiación: \\ ninguna declarada. \\ Aprobación ética: \\ La investigación fue considerada una \\ investigación sin riesgo. Los autores \\ declaran que los procedimientos seguidos \\ se realizaron de acuerdo a las normas \\ éticas de la Asociación Médica Mundial y \\ la Declaración de Helsinki. \\ Conflicto de intereses: \\ ninguna declarada. \\ Procedencia y revisión por pares: \\ revisado por pares. \\ Recibido el: 25/07/2018. \\ Aceptado el: 27/08/2018.
}




\begin{abstract}
In the context of the pre-summit and the VII Ibero-American Family Medicine Summit, held in Cali, Colombia on March 13 and 14, 2018, the research group and the Ibero-American Family Medicine Network (IBIMEFA) with the objective of identifying the perceptions of family doctors graduated and in process of formation of Ibero-America, on the conditions they have to develop applied research to the territory, carried out an exploratory, descriptive, cross-sectional study in the member countries of the Ibero-American Confederation of Family Medicine (CIMF)). For the collection of information, a survey was designed with 51 items, whose content was validated by the working group. The instrument was stored in Google Drive and its dissemination through virtual media. A response was obtained from 277 people, representatives from 16 countries of lbero-America in which it was found that there is interest and potential within the group of the IBIMEFA Network, to carry out studies with the research focus applied to the territory, although time difficulties must be overcome and funding to achieve it, as well as finding strategies that allow cooperative work to consolidate the Network.
\end{abstract}

Keywords: Family Practice; Health Determinants; Territory; Research

\title{
Resumo
}

No contexto da pré-cúpula e da VII Cúpula Ibero-Americana de Medicina de Família, realizada em Cali, Colômbia, em 13 e 14 de março de 2018 , o grupo de pesquisa e a Rede Ibero-Americana de Medicina Familiar (IBIMEFA) com o objetivo de identificar as percepções de médicos de família formados e em processo de formação da Ibero-América, sobre as condições que têm para desenvolver pesquisa aplicada ao território, realizaram um estudo corte-transversal, descritivo, e exploratório, nos países membros da Confederação Iberoamericana da Medicina Famíliar (CIMF)). Para a coleta de informações, foi elaborada um questionario com 51 itens, cujo conteúdo foi validado pelo grupo de trabalho. O instrumento foi armazenado no Google Drive e sua divulgação foi feita através de mídia virtual. Foi obtida resposta de 277 pessoas, representantes de 16 países da lbero-América e se verificou que há interesse e potencial dentro do grupo da Rede IBIMEFA, para realizar estudos com o foco de pesquisa aplicada ao território, ainda que se deva superar dificuldades relacionadas ao tempo e ao financiamento para conseguir realiza-la, bem como encontrar estratégias que permitam o trabalho cooperativo para consolidar a Rede.

Palavras-chave: Medicina de Família e Comunidade; Determinantes Sociais; Territorio; Pesquisa

\section{Introducción}

El fortalecimiento de la investigación en Medicina Familiar y Comunitaria ha sido uno de los ejes de trabajo en todas las cumbres convocadas por la Confederación Iberoamericana de Medicina Familiar (CIMF) ${ }^{1,2}$ Con motivo de la VII Cumbre Iberoamericana - "Cuarenta Años de Alma-Ata. Medicina Familiar y Salud Familiar Un Camino para la paz" en Cali-Colombia (2018), se integró como uno de los seis temas de análisis el concepto de Investigación aplicada al territorio. El tema es nuevo y no está claramente definido, aunque se puede caracterizar por dar relevancia al contexto territorial y de la población en diferentes procesos sociales, dentro de los cuales está la salud. Inicialmente, con el fin de tener un marco conceptual que permita a los lectores una comprensión similar del término "Territorio", se exponen algunas definiciones relacionadas con el tema.

Cuando se habla de territorio se entiende como el espacio geográfico que cuenta con límites y dimensiones de identidad, político-administrativas. ${ }^{3}$ En este coexisten subsistemas naturales y sociales que se modifican uno a otro. ${ }^{3}$ De otro lado los determinantes sociales en salud son "las circunstancias donde las personas nacen, crecen, viven, trabajan y envejecen, incluido el sistema de salud". ${ }^{4}$ Relacionando estos dos conceptos, se espera que más allá de dimensiones geográficas, se reconozca la interacción sujeto-objeto, la relación de la dimensión de salud-enfermedad, así como las interacciones de los determinantes sociales de la salud con el territorio. ${ }^{3-5}$ De esta manera, la investigación aplicada al territorio nos plantea el reto de definir problemas, objetivos, metodología, recolección y análisis de datos que permitan documentar esta relación. Idealmente se debería buscar desarrollar estos procesos con la comunidad de tal forma que permita el empoderamiento de su territorio y reconozca el carácter transformador de la realidad que toda intervención entraña y el compromiso de las mismas con el cuidado de la vida. 
Teniendo en cuenta este concepto amplio de territorio que comprende las condiciones de vida y por supuesto la suma de estos como un tejido dentro del que se presentan desigualdades e inequidades que repercuten en la salud de las personas, familias y comunidades, ${ }^{6}$ el médico familiar debe reconocer estos contextos para el desarrollo de modelos de atención, de gestión y de participación de la comunidad. La valoración de estudios orientados al territorio y al contexto puede profundizar el análisis desde un enfoque integral (biosicosocial) y nos anima a superar las barreras para la investigación desde esta perspectiva. ${ }^{7}$

\section{Relación entre Medicina Familiar y Comunitaria y los contextos poblacionales y de territorios}

La Atención Primaria en Salud (APS) entendida como estrategia "para organizar los sistemas de atención a la salud y la sociedad"8 comprende no solo los servicios de salud, también cuenta con el trabajo intersectorial y la participación comunitaria. Dentro de las características de la organización para la implementación de la APS, 9,10 se debe tener una puerta de entrada resolutiva donde está el principal escenario de actuación del especialista en medicina familiar; de otro lado y como bien lo expresaron Anderson y colaboradores ${ }^{11}$ en el marco de la $\vee$ Cumbre Iberoamericana de Medicina Familiar:

"Los médicos de familia son médicos especialistas en la prestación de cuidados de salud personalizados

y continuados, centrados en la persona, independientemente de la edad, sexo o afección, integrando los factores físicos, psicológicos, sociales, culturales y existenciales involucrados en el proceso salud-enfermedad. Prestan cuidados a los individuos según el contexto familiar, comunitario, cultural de los mismos, y tienen una responsabilidad profesional por su comunidad. Desempeñan su papel promoviendo la salud, previniendo la enfermedad y prestando cuidados asistenciales, de acompañamiento clínico, cuidados paliativos, y lo hacen de acuerdo las necesidades de salud, los recursos disponibles en la comunidad. Deben aún responsabilizarse por el desarrollo y mantenimiento de sus aptitudes, valores y equilibrio personales, como base para la prestación de cuidados efectivos y seguros. La Atención Primaria en Salud (APS) es el terreno de actuación fundamental del Médico de Familia, mientras que la Medicina Familiar es la herramienta clave para el desarrollo pleno de la APS."11

Barbara Starfield ha definido algunas características que debe cumplir la puerta de entrada del sistema de salud, para ser coherente con una organización de servicios basada en la APS ${ }^{10}$ que incluye cuatro atributos esenciales: el primer contacto, la continuidad, la integralidad (o extensión) y la coordinación; y tres atributos secundarios el enfoque familiar, la orientación comunitaria y la competencia cultural. ${ }^{12}$ Estas características son coherentes con el concepto expresado por Anderson y colaboradores ${ }^{11}$ sobre la forma de actuar de los médicos de familia, en el que relacionan la prestación centrada en la persona con el contexto familiar, comunitario y cultural de los mismos y la responsabilidad profesional por su comunidad.

Así surge la inquietud acerca de las posibilidades que puede tener el especialista en medicina familiar para realizar investigación aplicada al territorio y como se puede relacionar con tres áreas en las que se desenvuelven estos profesionales, como son las áreas: clínica, de gestión y formación del talento humano. 


\section{Objetivo general}

Identificar las percepciones de médicos familiares graduados y en proceso de formación de Iberoamérica, sobre las condiciones que tienen para desarrollar investigación aplicada al territorio.

\section{Método}

Para alcanzar el objetivo propuesto, se realizó un estudio exploratorio, descriptivo, de corte transversal en el que participaron médicos familiares (graduados y en formación) de los países miembros de la Confederación Iberoamericana de Medicina Familiar (CIMF).

La recolección de la información se efectuó entre el 01 y el 31 de enero de 2018, mediante un instrumento tipo encuesta en línea, auto-administrada, diseñada por los investigadores y validada en su contenido por el grupo de trabajo, la misma recogió un total de 51 items agrupados de la siguiente forma:

- Primer grupo; once items dirigidos a establecer datos demográficos de los encuestados.

- Segundo grupo; siete items dirigidos a establecer la capacidad que puede tener el grupo para participar en investigación.

- Tercer grupo; diecisiete items dirigidos a establecer dentro del grupo la relación de su práctica clínica con los determinantes de la salud e investigación clínica que relacione la información de la práctica clínica del médico familiar con el abordaje biopsicosocial que hace la especialidad.

- Cuarto grupo; nueve items dirigidos a establecer la adscripción poblacional y la responsabilidad de especialistas en medicina familiar como una forma indirecta de mirar la posibilidad de realizar investigación de gestión en salud.

- Quinto grupo; siete items dirigidos a relacionar las experiencias prácticas formativas desde un abordaje biosicosocial, que permita inferir la formación en investigación aplicada en el territorio.

El cuestionario se editó en Google Drive y su divulgación se realizó a través de correo electrónico y redes sociales de médicos familiares representantes de los veinte países que integran la CIMF. El análisis estadístico univariado se realizó a través de SPSS versión 25.0. Las conclusiones y recomendaciones se consolidaron durante la VII Cumbre.

\section{Resultados}

\section{Características de los encuestados}

Se obtuvo la respuesta de 282 encuestados, se excluyeron 5 encuestas que reportaron nivel de formación en pregrado o formación en otro campo diferente a la medicina familiar. Los 277 restantes, fueron médicos familiares (graduados y en formación), representantes de 16 países de la región iberoamericana: Argentina, Bolivia, Brasil, Chile, Colombia, Costa Rica, Cuba, Ecuador, España, México, Panamá, Paraguay, Perú, República Dominicana, Uruguay y Venezuela (Tabla 1). Según su procedencia por subregiones de la CIMF, se distribuyeron en la forma siguiente: Región Andina 147 (53,06\%), Cono Sur 72 (25,99\%), Mesoamérica $57(20,57 \%)$ y Península Ibérica 1 (0,4\%). El número de colaboradores por país fue muy disímil. Por una parte Colombia registró $36,8 \%$ de encuestados, seguido de México y Chile con alrededor 
del 10,5\% cada uno, mientras que España, Panamá y Cuba tuvieron participación inferior al 1\%, seguido de Paraguay, Perú y República Dominicana con menos del 2\%. En los países restantes, la contribución osciló entre el $3,5 \%$ al $7,1 \%$.

Tabla 1. Características de los encuestados: País de Residencia, edad y sexo.

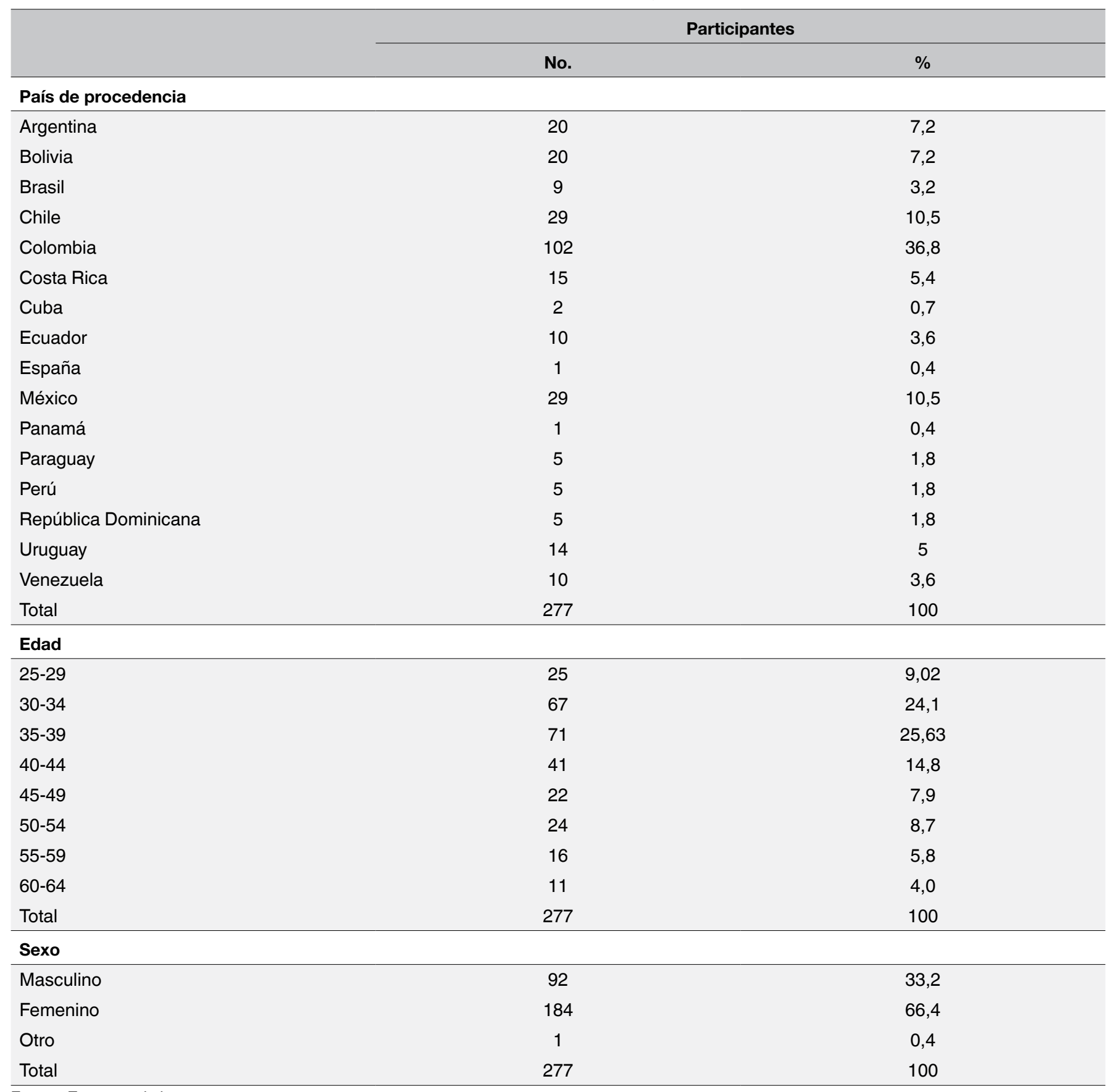

Fuente: Encuesta de los autores.

Las edades de los participantes estuvieron entre 25 y 64 años, con una media de 34,63 y moda de 36 años. El 50\% de los encuestados se ubicó en el grupo de 30 a 39 años. Predominó la participación de personas del género femenino $(66,4 \%)$. Hubo participación considerable de residentes $(17 \%)$, los cuales 
se incluyen en el análisis de datos, considerando que son profesionales médicos que trabajan en la APS, y que a pesar de estar en formación, están en contacto con la comunidad, los sistemas de salud y realizan investigación en territorio (Tablas 1 y 2).

Tabla 2. Características de los encuestados: Rol profesional, nivel de formación.

\begin{tabular}{|c|c|c|}
\hline & \multicolumn{2}{|c|}{ Participantes } \\
\hline & No. & $\%$ \\
\hline \multicolumn{3}{|l|}{ Rol profesional } \\
\hline Asistencial. & 86 & 31 \\
\hline Administrativo. & 11 & 4 \\
\hline Docente. & 19 & 6,9 \\
\hline En formación & 47 & 17 \\
\hline Docente/investigador & 4 & 1,4 \\
\hline Asistencial., Administrativo. & 13 & 4,7 \\
\hline Asistencial/docente & 63 & 22,7 \\
\hline Asistencial/Docente/Advo. & 32 & 11,5 \\
\hline Otros & 2 & 0,8 \\
\hline Total & 277 & 100 \\
\hline \multicolumn{3}{|l|}{ Nivel de formación } \\
\hline Médico Familiar & 230 & 83,0 \\
\hline Residente & 47 & 17,0 \\
\hline Total & 277 & 100 \\
\hline
\end{tabular}

Fuente: Encuesta de los autores.

En relación al rol profesional desempeñado por los encuestados (Tabla 2), el 41,9\% seleccionó un rol exclusivo, quedando distribuidos de la manera siguiente: $31 \%$ asistencial, $4 \%$ administrativo y $6,9 \%$ docente. Por otra parte, encontramos un $40,3 \%$ de médicos con roles mixtos, entre los que se destaca el asistencial/docente con $22,7 \%$ y un $11,5 \%$ que ejerce los tres roles asistencial/docente/administrativo. $\mathrm{Al}$ sumar los roles exclusivos y los mixtos prevaleció el rol asistencial con $70 \%$, seguido del docente con un $42,2 \%$ y el administrativo con un $19,9 \%$. El rol investigativo solo fue reportado por $1,4 \%$ de los participantes y estos provenían de Argentina Colombia, México y Uruguay.

Entre los que respondieron la encuesta, $62 \%$ respondió estar vinculado a la sociedad científica de su país, de los cuales, $7 \%$ también hacen parte de otra sociedad científica nacional o internacional. El 53\% de los encuestados solo maneja el idioma español; 43\% tiene algún grado de manejo del inglés y 10 del portugués. Solo $2,8 \%$ reportaron habilidad en el manejo de lenguas nativas.

En cuanto a la formación en el campo de la metodología en investigación (Tabla 3), prevalece la obtenida a través de formación no formal $(37,5 \%)$ como cursos, diplomados, seminarios entre otros, seguido por la formación obtenida durante el pregrado y la especialidad $(43,7 \%)$, maestrías $(10,5 \%)$ y doctorado (3,6\%). El 72,9 de los encuestados refirió que tienen menos de cinco años de experiencia en investigación, de los cuales, 34,7\% tienen menos de un año de experiencia. Es importante destacar que 7,9\% dijo tener más de 15 años de experiencia. Respecto a su rol en la investigación, de los 277 encuestados, 18,4\% respondió que sólo cuenta con la experiencia generada en su pregrado o no ha participado en los últimos 
Tabla 3. Características de los encuestados: Tipo de formación, años de experiencia y rol en investigación.

\begin{tabular}{lcc}
\hline & No & $\%$ \\
\hline Tipo de formación & & 37,5 \\
\hline Diplomado-cursos-seminarios & 104 & 43,7 \\
Formación de pregrado y en el postgrado de MF & 121 & 4,7 \\
Formación en alguna especialidad adicional & 13 & 10,5 \\
Maestrías & 29 & 3,6 \\
Doctorado & 10 & 100 \\
Total & 277 & 34,7 \\
\hline Años de experiencia & & 42,2 \\
\hline Menos de 1 año & 96 & 10,5 \\
1 a 5 & 117 & 4,7 \\
6 a 10 & 29 & 7,9 \\
11 a 15 & 13 & 100 \\
más de 15 & 22 & \\
Total & 277 & 14,8 \\
\hline Rol en la investigación & & 3,6 \\
\hline Tiene experiencia en pregrado & 41 & 18,8 \\
No participa en los últimos 12 meses & 10 & 26,7 \\
Docente en investigación & 52 & 33,2 \\
Investigador en formación & 74 & 2,9 \\
Investigador & 92 & 100 \\
Investigador de carrera & 8 & \\
Total & 277 & \\
\hline
\end{tabular}

Fuente: Encuesta de los autores.

doce meses en actividades de investigación (Tabla 3). Del total de encuestados (36,1\%), se consideran investigadores y docentes en investigación 18,8\%. Llama la atención la percepción del investigador en formación del 26,7\%.

En cuanto al tipo de investigación en que han participado el $43 \%$ refiere haber participado en investigación descriptiva, 33\% en investigación cualitativa, 16\% en investigación analítica y $3 \%$ en investigación experimental. Un 76\% de los encuestados había participado en más de uno de estos tipos de investigación.

Por otro lado, también se exploró información sobre las horas dedicadas a investigación, encontrándose que $10 \%$ de los encuestados destinaban 10 horas o más a la semana a la investigación y hubo personas que reportaron tener más de 10 publicaciones en los últimos 10 años.

\section{Opinión o percepción acerca de las condiciones que permitirían participar en investigación en el territorio en países de Iberoamérica}

En este apartado se recogió la percepción de cómo integrar el actuar del especialista en medicina familiar, en el que requiere no solo relacionar al individuo, sino comprender las condiciones de vida dentro de un contexto de desigualdades e inequidades en los territorios que habitan. 
La pregunta formulada al respecto: "En su práctica como especialista en medicina familiar; el lugar de trabajo y la organización para prestar el servicio, le facilita que en su abordaje clínico integre:" Las opciones incluyeron categorías de los determinantes sociales de la salud, y que tanto éstos son integrados en la atención médica (Tabla 4). Al tener en cuenta los que están de acuerdo o totalmente de acuerdo con este item, el 67,1\% considera que sí hay facilidades para la integración de las condiciones socioeconómicas, ambientales y culturales en el acto médico; $69,3 \%$ refirió que se integran los contextos familiares y sociales a la clínica; $61,7 \%$ dijo que integran a la clínica determinantes sociales como el desempleo, trabajo, vivienda, agricultura y alimentos y para el $51,8 \%$ se puede integrar a la clínica el entorno físico, ocio y cultura, servicios sociales y atención sanitaria.

Tabla 4. Integración de determinantes de la salud en la atención clínica.

\begin{tabular}{|c|c|c|c|c|c|c|c|c|c|c|c|c|}
\hline \multicolumn{13}{|c|}{ ¿Existe integración entre los siguientes determinantes de la salud y la atención clínica? } \\
\hline \multirow[t]{2}{*}{ Determinantes sociales } & \multicolumn{2}{|c|}{$\begin{array}{l}\text { Muy de } \\
\text { acuerdo }\end{array}$} & \multicolumn{2}{|c|}{ De acuerdo } & \multicolumn{2}{|c|}{ Indiferente } & \multicolumn{2}{|c|}{$\begin{array}{c}\text { En } \\
\text { desacuerdo }\end{array}$} & \multicolumn{2}{|c|}{$\begin{array}{c}\text { Muy en } \\
\text { desacuerdo }\end{array}$} & \multicolumn{2}{|c|}{ Total } \\
\hline & No & $\%$ & No & $\%$ & No & $\%$ & No & $\%$ & No & $\%$ & No & $\%$ \\
\hline $\begin{array}{l}\text { Condiciones socioeconómicas } \\
\text { ambientales y culturales }\end{array}$ & 61 & 22,0 & 125 & 45,1 & 16 & 5,8 & 34 & 22,0 & 41 & 14,8 & 277 & 100 \\
\hline Contextos familiares y sociales & 84 & 30,3 & 108 & 39,0 & 21 & 7,6 & 21 & 7,6 & 43 & 15,5 & 277 & 100 \\
\hline $\begin{array}{l}\text { Desempleo, trabajo, vivienda, } \\
\text { agricultura y alimentos }\end{array}$ & 64 & 23,1 & 107 & 38,6 & 30 & 10,8 & 39 & 14,1 & 37 & 13,4 & 277 & 100 \\
\hline $\begin{array}{l}\text { Entorno físico, ocio y cultura, } \\
\text { servicios sociales, atención sanitaria }\end{array}$ & 58 & 20,9 & 103 & 37,2 & 32 & 11,6 & 40 & 14,4 & 44 & 15,9 & 277 & 100 \\
\hline
\end{tabular}

Fuente: Encuesta de los autores.

En relación a "..si la organización de servicios facilita la integración clínica con los hábitos de vida y de trabajo", analizando a los que respondieron de acuerdo o totalmente de acuerdo, $73,29 \%$ percibe que se facilita la relación clínica con los hábitos de vida (alimentación, alcohol, tabaco, drogas, sueño); $59,57 \%$ considera que la organización de servicios facilita la integración clínica con las redes de apoyo y comunitarias; $71,84 \%$ estima que la organización de servicios facilita la integración clínica con las características personales de los individuos (edad, sexo, herencia genética) (Tabla 5).

Tabla 5. La organización de servicios de salud y los factores que facilitan la integración clínica.

\begin{tabular}{llllllllllll}
\hline \multicolumn{1}{c}{ ¿Hay relación entre la organización de los servicios y la facilidad de integración de los siguientes determinantes } \\
de la salud en la clínica?
\end{tabular}


Cabe anotar que $50 \%$ de los encuestados perciben que hay información sobre investigaciones relacionadas con determinantes de la salud, contextos familiares, sociales y comunitarios como parte del abordaje integral. Sin embargo, 52,63\% considera que aunque existen políticas en esta área, las mismas no son suficientes para apoyar la ejecución de investigaciones orientadas a los determinantes sociales o a los territorios. Es de resaltar que sólo el 30,3\% de los encuestados tiene la percepción de que las políticas actuales en sus países apoyan estos procesos investigativos; De hecho, la percepción de la población encuestada de poco a nada de apoyo en este tipo de investigación es predominante en países como Bolivia (70\%), México (55\%), Venezuela (90\%), Paraguay (90\%) y Colombia (55\%).

Respecto a la percepción de factores que influyen en el fortalecimiento de la investigación que relacione los determinantes de la salud con la clínica, $51,27 \%$ están muy de acuerdo y de acuerdo que existen obstáculos, solo $36,46 \%$ están de acuerdo o muy de acuerdo en que hay interés por realizar este tipo de investigación (Tabla 6). Frente a la percepción de factores que pueden influenciar el fortalecimiento de los procesos investigativos, se encuentra que $37,19 \%$ está muy de acuerdo y de acuerdo en que existen grupos dirigidos a este tipo de investigación en sus respectivos países.

Tabla 6. Percepción de factores que influyen en el fortalecimiento de la investigación.

\begin{tabular}{|c|c|c|c|c|c|c|c|c|c|c|c|c|}
\hline \multirow[t]{2}{*}{ Factores que influyen } & \multicolumn{2}{|c|}{$\begin{array}{l}\text { Muy de } \\
\text { acuerdo }\end{array}$} & \multicolumn{2}{|c|}{ De acuerdo } & \multicolumn{2}{|c|}{ Indiferente } & \multicolumn{2}{|c|}{$\begin{array}{c}\text { En } \\
\text { desacuerdo }\end{array}$} & \multicolumn{2}{|c|}{$\begin{array}{c}\text { Muy en } \\
\text { desacuerdo }\end{array}$} & \multicolumn{2}{|c|}{ Total } \\
\hline & No & $\%$ & No & $\%$ & No & $\%$ & No & $\%$ & No & $\%$ & No & $\%$ \\
\hline Obstáculos para la investigación & 35 & 12,64 & 107 & 38,63 & 50 & 18,05 & 58 & 20,94 & 27 & 9,75 & 277 & 100 \\
\hline Interés por realizar investigación & 18 & 6,50 & 83 & 29,96 & 62 & 22,38 & 87 & 31,41 & 27 & 9,75 & 277 & 100 \\
\hline Grupos de investigación identificables & 15 & 5,42 & 88 & 31,77 & 57 & 20,58 & 84 & 30,32 & 33 & 11,91 & 277 & 100 \\
\hline
\end{tabular}

Fuente: Encuesta de los autores.

\section{Medicina Familiar en el territorio y la gestión en salud}

La percepción de la existencia de adscripción poblacional es principalmente por divisiones políticoadministrativas, con un promedio de $40 \%$ para todos los países, así como la adscripción por aseguramiento de entidades público-privadas que en promedio es del 35\% (Tabla 7). Sin embargo hay una percepción importante de que no hay adscripción poblacional, cercana al $20 \%$ en promedio. La percepción de adscripción por las divisiones político-administrativas es mayor en Argentina, Brasil, Costa Rica, Cuba, Ecuador, España, Panamá y Uruguay. La percepción de adscripción por aseguramiento predomina en Bolivia, Colombia, México y Perú. En Venezuela predominó la percepción de que no hay una adscripción poblacional. Esta percepción también tuvo por lo menos un $20 \%$ o más en países como Bolivia, Colombia, Ecuador y Uruguay (Tabla 7).

\section{Medicina familiar en el territorio y la formación del talento humano}

Respecto a la formación se preguntó si ¿Las experiencias formativas para especialistas en medicina familiar les permiten tener desarrollar un enfoque integral, biopsicosocial, familiar y comunitario? En 85\% de los encuestados la percepción predominante fue que sí permite tener un enfoque integral biopsicosocial, 
Tabla 7. Percepción del tipo de adscripción poblacional.

\begin{tabular}{|c|c|c|c|c|c|c|c|c|c|c|c|}
\hline \multirow[t]{2}{*}{ País } & \multirow[t]{2}{*}{ Total } & \multicolumn{2}{|c|}{$\begin{array}{l}\text { No sabe/No } \\
\text { responde }\end{array}$} & \multicolumn{2}{|c|}{ Aseguramiento } & \multicolumn{2}{|c|}{$\begin{array}{c}\text { Distribución } \\
\text { cultural }\end{array}$} & \multicolumn{2}{|c|}{$\begin{array}{l}\text { Divisiones político } \\
\text { administrativa }\end{array}$} & \multicolumn{2}{|c|}{$\begin{array}{c}\text { No hay adscripción } \\
\text { poblacional }\end{array}$} \\
\hline & & No & $\%$ & No & $\%$ & No & $\%$ & No & $\%$ & No & $\%$ \\
\hline Argentina & 20 & 2 & 10 & 1 & 5 & 2 & 10 & 13 & 65 & 2 & 10 \\
\hline Bolivia & 20 & 0 & 0 & 10 & 50 & 0 & 0 & 6 & 30 & 4 & 20 \\
\hline Brasil & 9 & 0 & 0 & 0 & 0 & 0 & 0 & 9 & 100 & 0 & 0 \\
\hline Chile & 29 & 1 & 3 & 1 & 3 & 1 & 3 & 24 & 83 & 2 & 7 \\
\hline Colombia & 102 & 3 & 3 & 56 & 55 & 1 & 1 & 11 & 11 & 31 & 30 \\
\hline Costa Rica & 15 & 0 & 0 & 2 & 13 & 0 & 0 & 13 & 87 & 0 & 0 \\
\hline Cuba & 2 & 0 & 0 & 0 & 0 & 0 & 0 & 2 & 100 & 0 & 0 \\
\hline Ecuador & 10 & 0 & 0 & 1 & 10 & 1 & 10 & 6 & 60 & 2 & 20 \\
\hline España & 1 & 0 & 0 & 0 & 0 & 0 & 0 & 1 & 100 & 0 & 0 \\
\hline México & 29 & 0 & 0 & 16 & 55 & 1 & 3 & 7 & 24 & 5 & 17 \\
\hline Panamá & 1 & 0 & 0 & 0 & 0 & 0 & 0 & 1 & 100 & 0 & 0 \\
\hline Paraguay & 5 & 1 & 20 & 0 & 0 & 2 & 40 & 2 & 40 & 0 & 0 \\
\hline Perú & 5 & 0 & 0 & 3 & 60 & 0 & 0 & 2 & 40 & 0 & 0 \\
\hline República Dominicana & 5 & 0 & 0 & 2 & 40 & 1 & 20 & 2 & 40 & 0 & 0 \\
\hline Uruguay & 14 & 0 & 0 & 2 & 14 & 0 & 0 & 9 & 64 & 3 & 21 \\
\hline Venezuela & 10 & 0 & 0 & 2 & 20 & 0 & 0 & 2 & 20 & 6 & 60 \\
\hline Total & 277 & 7 & 3 & 96 & 35 & 9 & 3 & 110 & 40 & 55 & 20 \\
\hline
\end{tabular}

Fuente: Encuesta de los autores.

con ligeras variaciones entre los diferentes países, sólo México y República Dominicana se ubicaron por debajo del 65\% (Tabla 8). También se interrogó si ¿Las experiencias formativas (escenarios de práctica) les facilitan esta comprensión? En la región predomina la percepción de que la formación sí les permite comprender el contexto de la población y el territorio en $57 \%$ de los casos, opinión que predominó en Argentina, Bolivia, Brasil, Chile, Costa Rica, Cuba Ecuador, España Paraguay Uruguay y Venezuela. Los países que no tuvieron esta percepción fueron Panamá, Perú y República Dominicana. En Colombia y México las opiniones estuvieron divididas (Tabla 8).

\section{Discusión y Conclusiones}

Se trata de una investigación exploratoria que da continuidad a un proceso que ha seguido la CIMF a través de las diferentes Cumbres, con sus respectivos grupos de trabajo (incluyendo IBIMEFA), con los cuales, han buscado fomentar el desarrollo de redes de investigación e investigadores de alto nivel en el campo de la Medicina Familiar. ${ }^{13,14}$

Respecto a la participación en la encuesta y las características del grupo se puede comentar lo siguiente:

Fue una encuesta abierta y es importante mencionar que la participación de especialistas en medicina familiar mejoró respecto a los 114 participantes reseñado por Serrudo y otros ${ }^{14}$ en 2016. La mayoría de los encuestados tienen experiencia en investigación, como parte de sus funciones como formadores en la especialidad de medicina familiar y están ligados en su trabajo a universidades; sólo el 14,1\% tiene formación de maestría y doctorado. 
Tabla 8. Experiencias formativas y desarrollo de competencias en abordaje integral.

\begin{tabular}{|c|c|c|c|c|c|c|c|c|c|}
\hline \multicolumn{10}{|c|}{$\begin{array}{l}\text { ¿Las experiencias formativas para especialistas en medicina familiar les permiten tener desarrollar un enfoque integral, } \\
\text { biopsicosocial, familiar y comunitario? }\end{array}$} \\
\hline \multirow{3}{*}{ País } & \multirow{3}{*}{ Total } & \multicolumn{4}{|c|}{$\begin{array}{l}\text { La formación permite tener un enfoque } \\
\text { integral biopsicosocial }\end{array}$} & \multicolumn{4}{|c|}{$\begin{array}{l}\text { La formación permite comprender a la } \\
\text { población en contexto con su territorio }\end{array}$} \\
\hline & & \multicolumn{2}{|c|}{ NO } & \multicolumn{2}{|c|}{$\mathrm{Si}$} & \multicolumn{2}{|c|}{ NO } & \multicolumn{2}{|c|}{ SI } \\
\hline & & No & $\%$ & No & $\%$ & No & $\%$ & No & $\%$ \\
\hline Argentina & 20 & 0 & 0 & 20 & 100 & 7 & 35 & 13 & 65 \\
\hline Bolivia & 20 & 1 & 5 & 19 & 95 & 7 & 35 & 13 & 65 \\
\hline Brasil & 9 & 1 & 11 & 8 & 89 & 2 & 22 & 7 & 78 \\
\hline Chile & 29 & 2 & 7 & 27 & 93 & 11 & 38 & 18 & 62 \\
\hline Colombia & 102 & 19 & 19 & 83 & 81 & 49 & 48 & 53 & 52 \\
\hline Costa Rica & 15 & 0 & 0 & 15 & 100 & 5 & 33 & 10 & 67 \\
\hline Cuba & 2 & 0 & 0 & 2 & 100 & 0 & 0 & 2 & 100 \\
\hline Ecuador & 10 & 0 & 0 & 10 & 100 & 4 & 40 & 6 & 60 \\
\hline España & 1 & 0 & 0 & 1 & 100 & 0 & 0 & 1 & 100 \\
\hline México & 29 & 11 & 38 & 18 & 62 & 15 & 52 & 14 & 48 \\
\hline Panamá & 1 & 0 & 0 & 1 & 100 & 1 & 100 & 0 & 0 \\
\hline Paraguay & 5 & 1 & 20 & 4 & 80 & 1 & 20 & 4 & 80 \\
\hline Perú & 5 & 1 & 20 & 4 & 80 & 4 & 80 & 1 & 20 \\
\hline República Dominicana & 5 & 2 & 40 & 3 & 60 & 4 & 80 & 1 & 20 \\
\hline Uruguay & 14 & 3 & 21 & 11 & 79 & 4 & 29 & 10 & 71 \\
\hline Venezuela & 10 & 1 & 10 & 9 & 90 & 4 & 40 & 6 & 60 \\
\hline Total & 277 & 42 & 15 & 235 & 85 & 118 & 43 & 159 & 57 \\
\hline
\end{tabular}

Fuente: Encuesta de los autores.

En el trabajo de Fernández y otros, señalan la dificultad de investigar en América Latina, para 2011 la región aportaba 3,8\% de los investigadores de tiempo completo del mundo. ${ }^{12}$ Esta proporción no ha cambiado mucho, a pesar del aumento del presupuesto global para la investigación, en 2015 oscilaba en 3,9\% y siendo financiada mayormente por las universidades. La inversión en ciencia y tecnología en América Latina se concentra en tres países Brasil, con el 64\%; México, el 17\%, y Argentina, el $7 \% .{ }^{15}$

En la encuesta, 76,9\% refirieron tener experiencia menor a cinco años dedicada a investigación y $26,7 \%$ se identificaron como investigadores en formación, lo que implica que el grupo tiene un potencial para mejorar en este aspecto. Existe la necesidad de incrementar y perfeccionar la formación en investigación durante la residencia y de investigadores formales en las áreas de Medicina Familiar y APS, que puedan desarrollar investigación en territorio de alto nivel y no sólo como prácticas formativas en investigación a través de las tesis de grado. Se deben impulsar estrategias efectivas que mejoren las competencias en esta área para posgraduados, a través de programas de formación y capacitación continuas, actividades de investigación pre o trans congresos o incluso las pasantías en investigación referidas por Serrudo y colaboradores. ${ }^{14}$ Que se tenga en el grupo, personas con más de 10 publicaciones en la última década es una fortaleza, que puede guardar relación con el aumento de publicaciones observado en medicina familiar en los últimos años. ${ }^{13}$ 
Respecto a la percepción que se tienen acerca de si la organización de servicios de salud en los diferentes países, permite articular los determinantes de la salud de la población a los roles clínico, de gestión y de formación del médico de familia. La percepción fue que alrededor de tres cuartas partes de los médicos (73.29\%), consideraron que se puede relacionar la práctica clínica a los determinantes de la salud, principalmente en lo relacionado con hábitos de vida (alimentación, ingesta de alcohol, tabaco, drogas, sueño) y $71,84 \%$, con las características personales de los individuos (edad, sexo, herencia genética). Estos hallazgos son preocupantes, ya que lo esperado sería que el cien por ciento de los médicos familiares tuvieran la percepción (y las competencias) de que los determinantes sociales no sólo se pueden relacionar con la práctica clínica, sino que se deben integrar en el tan mencionado abordaje integral, biopsicosocial, holístico, etc., que realiza el médico familiar, ya que es parte del perfil esencial que no puede perder. Será interesante profundizar las características del perfil de formación y los países de procedencia de los profesionales que no lo consideraron posible, para en todo caso, recomendar algún tipo de actividad formativa compensatoria de esas deficiencias. Por otro lado, estos resultados también permiten vislumbrar un potencial para realizar investigación dirigida a comprender mejor como los médicos familiares pueden abordar e impactar en los determinantes de la salud y relacionarlas con el territorio.

Es posible que la percepción de no adscripción de la población fija por aseguramiento, área geográfica, afinidad o por el mecanismo que sea, además de la falta de continuidad de la atención por el mismo motivo; son limitantes para la realización de investigación en territorio. Además de las limitaciones que puede entrañar un modelo de salud desorganizado y que generalmente no está basado en Medicina Familiar o Salud Familiar. La percepción de que existen estas barreras para realizar investigación, da una idea clara del esfuerzo que implica transformar esta realidad en muchos de los países de la región.

En el trabajo de Fernández y otros, se hace mención de que existen tres limitantes importantes para realizar investigación en América Latina: Falta de financiamiento, la no participación de medicina familiar al no ser considerada como disciplina en algunos países y finalmente, la percepción de que la investigación en medicina familiar es de bajo impacto, por no generar patentes, innovaciones en patologías de alto impacto económico, etc. ${ }^{13}$ En este sentido, la mayoría de los encuestados refirió experiencias en investigación descriptiva (43\%) y cualitativa (33\%) que muchas veces son consideradas de bajo impacto y muy pocos en investigación analítica (16\%) y experimental (3\%) que tienen una mejor consideración.

El potencial que existe en el grupo y la identificación de las limitaciones que existen en el contexto, la importancia del aporte que puede realizar nuestra especialidad en la investigación en el territorio nos anima a seguir explorando sobre preguntas que surgieron en las mesas de trabajo convocadas por CIMF durante la VII Cumbre Iberoamericana de Medicina Familiar en Cali, Colombia y no desfallecer en esfuerzos para lograr trabajos cooperativos que resuelvan dudas como las siguientes: ¿Qué investigaciones clínicas podemos realizar que nos distingan e identifiquen la concepción integral de nuestra especialidad? ¿Cómo podemos destacar la importancia de la adscripción poblacional para la gestión de recursos en los territorios a cargo de los equipos de salud? ¿Cómo podemos mejorar las experiencias de formación en este tipo de investigaciones para los estudiantes? ¿Realmente los médicos familiares están empoderados y pueden aportar a la investigación en el territorio desde la medicina familiar? 
Durante las mesas de análisis realizadas durante la VII Cumbre convocada por CIMF, a propósito del diagnóstico situacional presentado en este documento, los representantes de los países participantes determinaron por consenso hacer las siguientes recomendaciones generales para impulsar la investigación en territorio desde la perspectiva de la Medicina Familiar:

1. Contribuir en el fortalecimiento de la Red IBIMEFA para la integración de investigadores de la Región, así como en la identificación y divulgación de oportunidades de formación; financiación; generación de información y reuniones periódicas para protocolos concretos.

2. Mejorar los canales de comunicación de las tesis/trabajos de grado/trabajos de campo que realizan los estudiantes/residentes para conocimiento de la Región, en pro del seguimiento de los resultados como del conocimiento de este tipo de investigación y establecer repositorios de tesis/investigaciones y bases de datos para definición de líneas de investigación.

3. Incentivar y promover la investigación clínica y epidemiológica con factor diferencial, con fundamentos y principios de medicina familiar (uso de herramientas de Salud y Medicina Familiar) y retomar contacto con los subgrupos de cumbres anteriores, así como con los coordinadores de grupos de la Red IBIMEFA, identificando posibilidades concretas de financiación.

\section{Limitantes}

La muestra no es significativa, no obstante denota un aumento en la participación en este tipo de encuestas realizadas en las Cumbres Iberoamericanas de Medicina Familiar y permite un acercamiento al interés y posibilidades que tenemos sobre el tema. Las percepciones registradas tienen un valor cualitativo importante dado que es la primera vez que se explora este tema relacionando el concepto de investigación en el territorio y la medicina familiar. También amplía la información disponible sobre la medicina familiar en su relación con la investigación en el territorio y constituye una línea base para el trabajo a seguir por parte de la CIMF. La participación mayoritaria de Colombia, país sede de la Cumbre, y por ende, con mayor participación (36,5\%), puede sesgar la información a conceptos que predominen en ese país. Los datos registrados tienen validez interna para el grupo de personas que diligenciaron la encuesta. Dentro de las posibilidades de mejora, está promover que este tipo de información es valiosa, por lo que debemos lograr una participación más significativa de todos los países de la región y ser más precisos en las variables que queremos estudiar. Este tipo de trabajos descriptivos solo podrá mostrar su valía en la medida que se le dé continuidad para poder comparar su evolución en el tiempo.

\section{Agradecimientos}

A Josep Lluís Piñol - QEPD (Miembro, Confederación Iberoamericana de Medicina Familiar), Lilia González Cárdenas (Miembro, Confederación Iberoamericana de Medicina Familiar) y Jacqueline Ponzo (Coordinadora de la Red IBIMEFA) por su valioso acompañamiento y consejos para la realización de este trabajo, así como a Daniel Ulate y José Manuel Ramírez Aranda, por su contribución al diseño de la encuesta. 


\section{Referencias}

1. Herrera JA. La investigación en medicina de familia en el siglo XXI. Aten Primaria. 2008; 40 (9): 435-6.

2. Rubinstein A. Investigación en la práctica de la medicina familiar: ¿una causa perdida o un desafío pendiente. Colomb Med. $2012 ; 43$ (1): 103-107.

3. Rodríguez-Páez FG, Vaca Hortúa DA, Manrique Méndez LV. Revisión de los conceptos de territorio, población y salud en el contexto colombiano. Cien tecnol salud vis ocul. 2012; 10 (2): 79-92. http://dx.doi.org/10.19052/sv.1437

4. World Health Organization. Determinantes de la Salud. [Internet] [Consultado: Diciembre 12 de 2017]. Disponible en: http://www.who. int/social_determinants/es/

5. Borde E, Torres-Tovar M. El territorio como categoría fundamental para el campo de la salud pública. Saúde Debate. 2017; 41 (spe2): 264-75. http://dx.doi.org/10.1590/0103-11042017s222

6. Iñiguez Rojas L. Territorio y contextos en la salud de la población. Revista Cubana de Salud Pública [Internet]. FapUNIFESP (SciELO); 2008 Mar;34(1):0-0. http://dx.doi.org/10.1590/S0864-34662008000100006

7. Macinko J, Montenegro H, Nebot Adell C, Etienne C. La renovación de la atención primaria de salud en las Américas. Revista Panamericana de Salud Pública [Internet]. FapUNIFESP (SciELO); 2007 Mar;21(2-3). http://dx.doi.org/10.1590/S1020-49892007000200003

8. Starfield B. Gervas J. Family Medicine Should Encourage Its Clinicians To Subspecialize: Negative Position In: Ideological Debates in Family Medicine, Nova Science Publishers, Inc, Editors: S.A. Buetow and T.W. Kenealy, Nova Science Publishers, 2007.

9. Organización Panamericana de la Salud (OPS) (2008). Redes integradas de servicios de salud. Conceptos, opciones de política, y hoja de ruta para su implementación en las Américas. Serie La renovación en la Atención Primaria de Salud en las Américas. Available from: http://www.paho.org/uru/index.php?option=com_docman\&view=download\&category_slug=publicaciones-sistemas-y-servicios-desalud\&alias=145-redes-integradas-de-servicios-de-salud-aps-n4\&ltemid=307

10. Villalbí JR, Pasarín M, Montaner I, Cabezas C, Starfield B. Evaluación de la atención primaria de salud. Atención Primaria [Internet]. Elsevier BV; 2003;31(6):382-5. http://dx.doi.org/10.1016/S0212-6567(03)70703-3

11. Anderson MIP, Rojas ML, Taureaux N, Cuba MS. Cobertura Universal en Salud, Atención Primaria y Medicina Familiar. Rev Bras Med Fam Comunidade. 2016;11(Suppl 1):4-30. http://dx.doi.org/10.5712/rbmfc11(1)1276

12. Berra, S. El estudio de las funciones de la Atención Primaria en Salud 2010; Available at: http://ciess. webs.fcm.unc.edu.ar/instrumentospcat-aps/. Accessed January 23, 2016.

13. Fernández MA, Rojas G, Irigoyen A, Roo JB. Producción y difusión del conocimiento en Medicina Familiar en Iberoamérica. Rev Bras Med Fam Comunidade. 2016;11(Suppl 1):71-87. http://dx.doi.org/10.5712/rbmfc11(1)1280

14. Serrudo ND, Ponzo J, Ramírez-Aranda JM, Argudo CH, Riveros MR, Vargas PV, et al. Investigación en Medicina Familiar y Comunitaria en Iberoamérica. Rev Bras Med Fam Comunidade. 2016;11(Suppl 2):64-74. http://dx.doi.org/10.5712/rbmfc11(0)1387

15. Albornoz M, Barrere R, Sokil J, Crespo JM, y Otros. El estado de la ciencia. Principales Indicadores de Ciencia y Tecnología Iberoamericanos/Interamericanos 2017.2017 Red de Indicadores de Ciencia y Tecnología - Iberoamericana e Interamericana - (RICYT). http://www.ricyt.org/files/Estado\%20de\%20la\%20Ciencia\%202017/El_Estado_de_la_Ciencia_2017_Completo.pdf 